Responses of a I ong-coi I pul se- modul at ed i nduct i on pl asma

\begin{tabular}{|l|l|}
\hline 著者 & $\begin{array}{l}\text { Paul K C. , Hossai n M M, Hashi mot o Y. , Tanaka } \\
\text { Yasunor i , Sakut a Tadahi ro }\end{array}$ \\
\hline $\begin{array}{l}\text { j our nal or } \\
\text { publ i cat i on t i t l e }\end{array}$ & I EEE Transact i on on Pl asma Sci ence \\
\hline vol une & 29 \\
\hline number & 2 \\
\hline page range & $326-334$ \\
\hline year & $2001-04-01$ \\
\hline URL & ht t p: //hdl . handl e. net /2297/1803 \\
\hline
\end{tabular}




\title{
Responses of a Long-Coil Pulse-Modulated Induction Plasma
}

\author{
K. C. Paul, Member, IEEE, M. M. Hossain, Student Member, IEEE, Y. Hashimoto, Y. Tanaka, and \\ Tadahiro Sakuta, Member, IEEE
}

\begin{abstract}
Radio-frequency inductively coupled plasma in a pulse modulated approach was generated by a MOSFET inverter supply of high electric efficiency. The plasma torch has an extremely long coil region of $153 \mathrm{~mm}$, which is an attractive feature for advanced materials processing, especially for better and more efficient vaporizing of solids. The operating conditions were: argon flow of 80 or $90 \mathrm{~L} / \mathrm{m}$ at atmospheric pressure; supply power of $30 \mathrm{~kW}$; and pulse on-time of $10 \mathrm{~ms}$ at $67 \%$ duty factor. Spectroscopic measurements were carried out to determine the temporal plasma properties, including the effects of shimmer current level (SCL) upon the spectral intensities. Additionally, a time-dependent two-dimensional numerical model was solved for the same operating conditions employed in the experiment to predict and compare the plasma properties. Pulsed plasma dissipation sustained for a minimum SCL of $43 \%$ for $80 \mathrm{~L} / \mathrm{m}$ gas flow-rate, and at any level below $43 \%$, the plasma disappeared. Temporal variation of argon line intensities at 751 and 763.5 $\mathrm{nm}$ is similar, though the upper level intensity of the former one was significantly stronger than the latter. Intensified change of intensity is found at lower SCL because of higher change in the coil current and, in turn, in the plasma power. The predicted intensity of the 751-nm argon line showed similar behavior to the experimental intensity though the response around the instant of on-pulsation is somewhat slower.
\end{abstract}

Index Terms-Duty factor, integrated radiation intensity, local thermodynamic equilibrium, pulse-modulated induction plasma, response times, shimmer current level, skin depth.

\section{INTRODUCTION}

$\mathbf{P}$ ULSATION in thermal plasma is a newer approach, which finds many importances in the applications of thermal plasma technology. The thermal plasmas are in extensive industrial and research applications, [1] and the trend is always pronouncing. So far, the applications of thermal plasmas are mostly of the steady-state operation. It is an anticipation

Manuscript received February 8, 2000; revised December 15, 2000. This work was supported in part through Special Coordination Funds for Promoting Science and Technology from the Science and Technology Agency of the Japanese Government and the Society of Non-Traditional Technology.

K. C. Paul is with the Power Group, Electrical and Computer Engineering Department, University of Toronto, Toronto ON, M5S 3G4 Canada (e-mail: khokan@power.ele.utoronto.ca).

M. M. Hossain is with the Department of Electrical and Electronic Engineering, Bangladesh Institute of Technology, Chittagong, Bangladesh (E-mail: 4129hoss@ect.kanazawa-u.ae.jp).

Y. Hashimoto was with the Department of Electrical and Electronic Engineering, Faculty of Engineering, Kanazawa University, Kanazawa 920-8667, Japan. He is now with Nippon Telegraph and Telephone Corporation, Tokyo 100-8116, Japan

Y. Tanaka and T. Sakuta are with the Department of Electrical and Electronic Engineering, Faculty of Engineering, Kanazawa University, Kanazawa 920-8667, Japan (e-mail: sakuta@t.kanazawa-u.ac.jp).

Publisher Item Identifier S 0093-3813(01)02891-0. that time domain control of thermal plasma will proliferate its application in diverged areas. Time-dependent control of plasma is capable of controlling both the plasma power and the species density with time, so that materials processing could be upgraded by controlling operation conditions.

Despite a huge application of thermal plasma technology in materials processing, some researchers haven been working to use this technology for fundamental study of high-pressure discharges [2], [3], which usually occur in a gas circuit breaker (GCB). Transient plasma gives an idea on different response times of plasma, and these would be representative of the so-called "time constants" of circuit breaker arcs. Please note that time constants are considered very important by a GCB engineer, because these values represent how fast a gas medium of GCB can respond with any transition in power systems.

Until today, there are a very few experimental revelations on plasma behavior for transient approach. Investigation on transient extinction conditions in an inductively coupled argon plasma, by Meubus [4]; power modulation (0.6-1.8 kW of plate power) effect on electron's temperature and density of an 18-mm diameter inductively coupled plasma (ICP), by De Regt et al. [5]; spectrochemical analyzes for modulation, by Fey et al. [6]; and coil current modulation effect upon the atomic argon line intensity at $751 \mathrm{~nm}$ by Sakuta et al. [7] and Ishigaki et al. [8], are some works to be noted.

A few reports on numerical predictions have also been found in the literature. These numerical models usually solve the conservative equations of mass, momentum, and energy simultaneously. Indeed, such numerical approach costs only a fraction of the cost required in any experimental approach and, thus, these models find substantial importance among researchers. Steadystate predictions are, however, enormous so far [9]-[14]. In the transient approach, one-dimensional model of Sakuta et al. [15], [16] to investigate time-dependent plasma properties, nonequilibrium and time-dependent behavior study by the model of Suekane et al. [17], and the two-dimensional (2-D) model of Mostaghimi et al. [18] are to be mentioned. The power profile model of time-dependent ICP code [18] is upgraded by Paul et $a l$. [19] for coil current input, since coil current modulation is more feasible.

In this paper, we report on the dynamic behavior of an argon radio-frequency (RF)-ICP for a special torch: a huge coil length of $153 \mathrm{~mm}$. This ten- or eight-turns coil length must improve materials processing, since any particle will be in plasma-contact for a longer time. Another feature of the setup is that the solid-state generator employed a metal oxide semiconductor field effect transistor (MOSFET), which has high electrical 


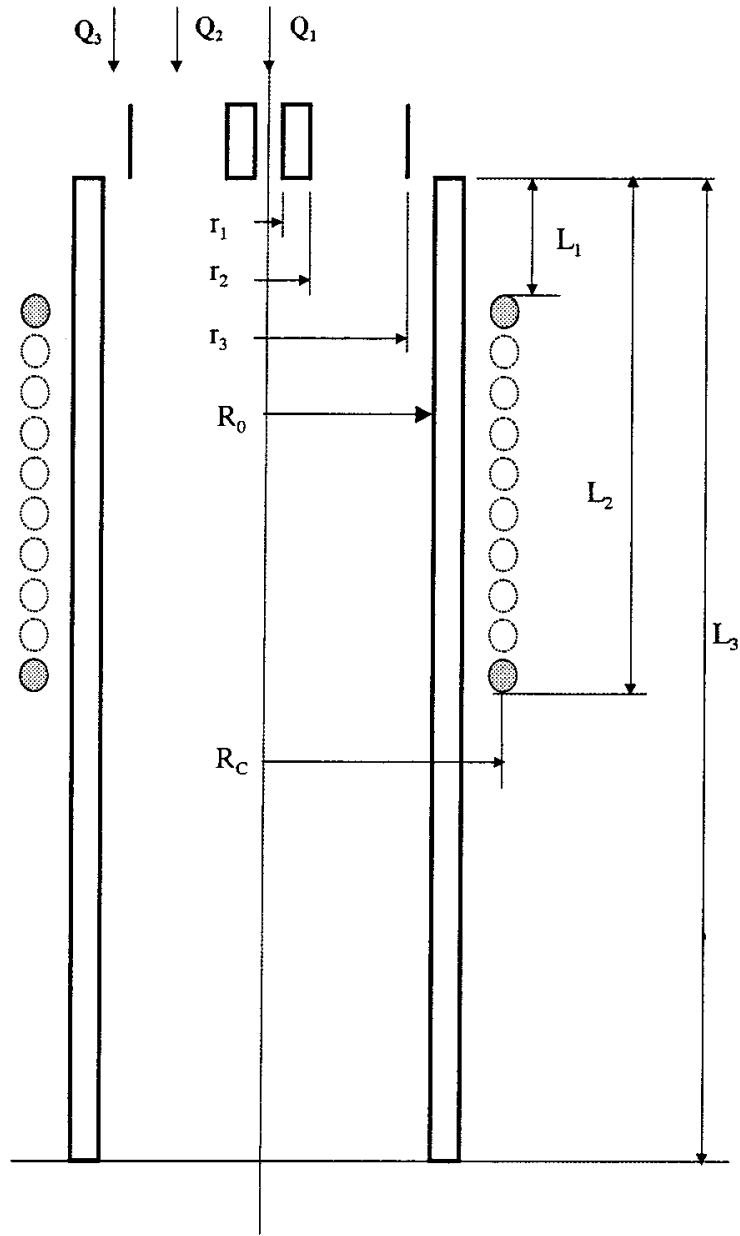

Fig. 1. Schematic geometry of the RF-ICP torch.

efficiency. The plasma was operated at a reactor pressure of 760 torr, supply power before pulsation of $30 \mathrm{~kW}$, total gas flow rate of 80 or $90 \mathrm{~L} / \mathrm{m}$ through the sheath channel, and pulsing signal of $10 \mathrm{~ms}$ on-time with $67 \%$ duty factor. Spectroscopic measurements are done to see the time-dependent behavior of plasma using two spectral lines of atomic argon at 751 and $763.5 \mathrm{~nm}$, respectively. The shimmer current level (SCL) effect upon the plasma behavior is determined both experimentally and theoretically using the spectral intensity of 751-nm line.

\section{EXPERIMENT AND MEASURING TECHNIQUE}

Schematics of the induction plasma torch are depicted in Fig. 1. The dimensions of the torch are listed in Table I. The torch has a standard configuration of two co-axial tubes of $370 \mathrm{~mm}$ in length, and it is surrounded by a water-cooled RF coil. The inner diameter of the inner quartz tube and the outer diameter of the outer pyrex tube are 70 and $95 \mathrm{~mm}$, respectively. The induction coil consists of ten or eight turns, and the length of the coil region is an extremely large $153 \mathrm{~mm}$, the longest in the world according to our best knowledge. The torch has three gas inlets (carrier, plasma and sheath), though we injected gas through the sheath channel only with swirl from the outer slots of the gas distributor. The setup used in the experiment for the measurement of temporal plasma fields is shown in Fig. 2. A solid state amplifier (HF0030W45MS, Fuji Electric Company,
Ltd.) was employed for the power supply. The inverter-type power source continuously supplies the electric power of 30 $\mathrm{kW}$ (maximum) with a nominal frequency of $450 \mathrm{kHz}$, and has a high matching efficiency of $85 \%$. The inverter source works by a MOSFET. Implementation of a MOSFET has the merits over other solid state devices for inverter are: 1) MOSFET has only one carrier (hole or electron), so that it responds fast; 2) it can be operated at high frequency around megahertz range; and 3 ) it is voltage-controlled, so that it is workable at a high power level. Transmission of the electrical power from the inverter to the plasma was made by a series $L C$ resonance circuit. The RF power was pulse modulated by imposing an external pulsed signal to achieve current pulsation; the rising time of the exciting current is as short as of $0.2 \mathrm{~ms}$. An optical system, with a spatial resolution of $1 \mathrm{~mm}$, was employed to obtain the plasma emission, which was transmitted through an optical fiber. Atomic argon line intensities at 751 and $763.5 \mathrm{~nm}$ were measured using a monochromator (JOBIN YVON HR-320) and detected by a photomultiplier (Hamamatsu R1104) of 70 $\mathrm{ns}$ response time. The detected signal was stored in a personal computer through a digitizer (Autonics APC 510) of a $50 \mathrm{~ns}$ per address minimum sampling time.

\section{SPECTROSCOPICALly MeAsured Results}

Fig. 3 shows a set of spectroscopically measured data for (a) pulsed signal intensity; (b) coil current; and (c) atomic argon line intensity. These results correspond to a $63.1 \% \mathrm{SCL}$, and $80 \mathrm{~L} / \mathrm{m}$ flow-rate. Other operating conditions are mentioned in Table I. The definition of the SCL and duty factor, $\eta$ are as of [7]

and

$$
\operatorname{SCL}[\%]=\frac{I_{\text {off }}}{I_{\text {on }}} \times 100
$$

$$
\eta[\%]=\frac{T_{\text {on }}}{T_{\text {on }}+T_{\text {off }}} \times 100
$$

where $I_{\text {on }}$ and $T_{\text {on }}$ are on-time current and time, and $I_{\text {off }}$ and $T_{\text {off }}$ are the on-time current and time, respectively.

The supply power was $30 \mathrm{~kW}$ (measured by the wattmeter installed upon the supply) while the plasma was continuous, or steady, without any pulse modulation. During pulse mode operation, the coil current was modulated periodically at a frequency of $67 \mathrm{~Hz}$ (10 ms on-time and $5 \mathrm{~ms}$ off-time, thus the period is $15 \mathrm{~ms}$ ). The input power to plasma during the pulse mode operation was not possible to measure, and, thus, unknown. It is seen from the coil current response [Fig. 3(b)], that the coil current did not respond instantaneously with the pulsed signal, but needed a time to follow. This time can be termed as response time of the coil current. The response time is found to be same at both pulse on- and off-times of $0.2 \mathrm{~ms}$. Reactive nature of the RF network is mainly responsible for such time-lagging response of the coil current. Radiation intensity of atomic argon line also responded with a time lag compared to the pulsed signal. This delayed response of the atomic argon line intensity is for two obvious reasons: 1) radiation intensity had a response time to follow the changes incurred by the pulse modulation and 2) delayed response of the coil current consequently delays the re- 
TABLE I

Torch Dimensions and Operating Conditions for the Constant Power Profile Case

\begin{tabular}{|c|c|}
\hline $\begin{aligned} \mathrm{R}_{1} & =1 \mathrm{~mm} \\
\mathrm{R}_{2} & =2 \mathrm{~mm} \\
\mathrm{R}_{3} & =34 \mathrm{~mm} \\
\mathrm{R}_{0} & =35 \mathrm{~mm} \\
\mathrm{R}_{\mathrm{c}} & =58 \mathrm{~mm} \\
\mathrm{~d}_{\text {coil }} & =14 \mathrm{~mm} \\
\text { Wall Thickness } & =5 \mathrm{~mm} \\
\text { No. of Coil Turns } & =10 \mathrm{or} 8 \\
\mathrm{f} & =450 \mathrm{kHz} \\
\text { Pulse on-time } & =10 \mathrm{~ms}\end{aligned}$ & $\begin{aligned} \mathrm{Q}_{1} & =0 \\
\mathrm{Q}_{2} & =0 \\
\mathrm{Q}_{3} & =80 \mathrm{or} 90 \text { slpm } \mathrm{Ar} \\
\mathrm{L}_{1} & =60 \mathrm{~mm} \\
\mathrm{~L}_{2} & =213 \mathrm{~mm} \\
\mathrm{~L}_{3} & =370 \mathrm{~mm} \\
\text { Pressure } & =760 \mathrm{Tor} \\
\text { Forward Power } & =30 \mathrm{~kW} \\
\text { Duty Factor, } \eta & =67 \% \\
\text { Pulse off-lime } & =5 \mathrm{~ms}\end{aligned}$ \\
\hline
\end{tabular}

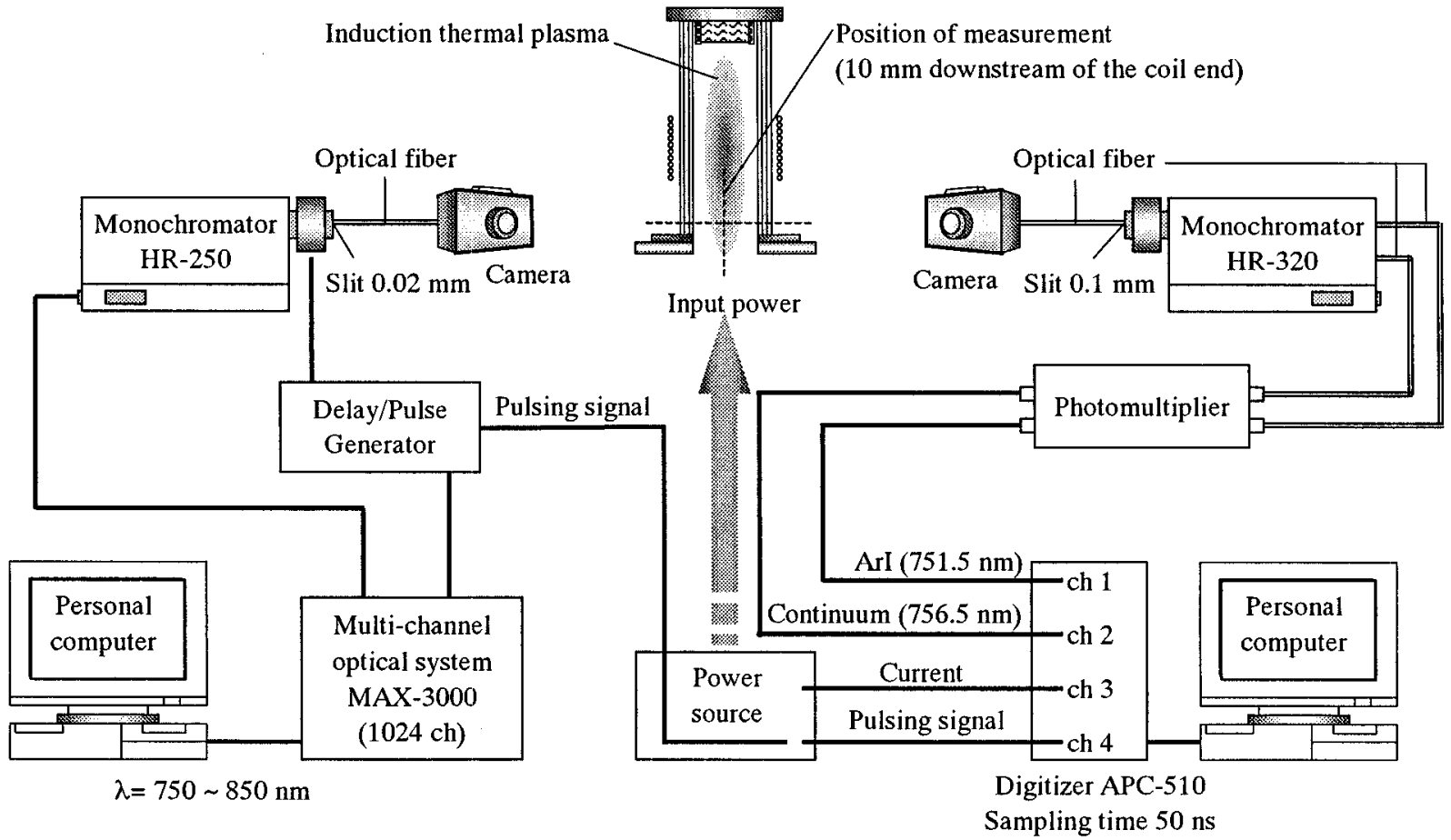

Fig. 2. Optical system used for the spectroscopic measurements.

sponse, since the radiation signal is, intrinsically, supposed to follow the input power or the coil current. The spectral intensity apparently attained steady-state value during on-time of the pulsed signal at about $6 \mathrm{~ms}$ after the transition. But it passed through complete transition during off-time. The response times of radiation intensity for on- and off-time modulations are found as 4 and $3 \mathrm{~ms}$, respectively. Definitions of the response times are explained clearly later in Fig. 12.

The SCL effect upon the radiation intensity of atomic argon line at $751 \mathrm{~nm}$ is depicted in Fig. 4. It is perceivable from the figure that the variation of radiation intensity becomes stronger with the decrease of SCL. The lower value of SCL means the lower current level during off-time of the pulsed signal. Since the upper current level during on-time is the same, the lower SCL, thus, causes a bigger current fluctuation by the pulse modulation. The bigger coil current fluctuation should make larger difference of plasma power levels between on-and off-times, which will eventually be responsible for a larger variation of the radiation intensity between the two levels. Another effect of SCL is that the radiation intensity takes a little longer time to respond with the pulsated change of the coil current for on-time. At lower SCL, more drop in the plasma temperature, as well as volume, happens during off-time, so that an additional time is required to raise plasma temperature or volume at a level equivalent to higher SCL. However, the response time of the argon line intensity did not vary with SCL considerably during pulse off-time, because the upper level of coil current during on-time is the same for all SCL's, so that the plasma state is similar to (though not exactly due to difference in plasma state during off-time) immediately before the off-pulsation.

Experiments were carried out for another set of operating conditions: $90 \mathrm{~L} / \mathrm{m}$ total flow rate; constant SCL of $62 \%$; 8 coil turns; and all other conditions were the same as in Table I. The time-dependent change of ArI line at three radial positions ( $r=$ $0,17.5 \mathrm{~mm}$, and $26.25 \mathrm{~mm}$ ) were measured at mid-coil region $(z=136.5 \mathrm{~mm})$ for two lines at 751 and $763.5 \mathrm{~nm}$, and are 

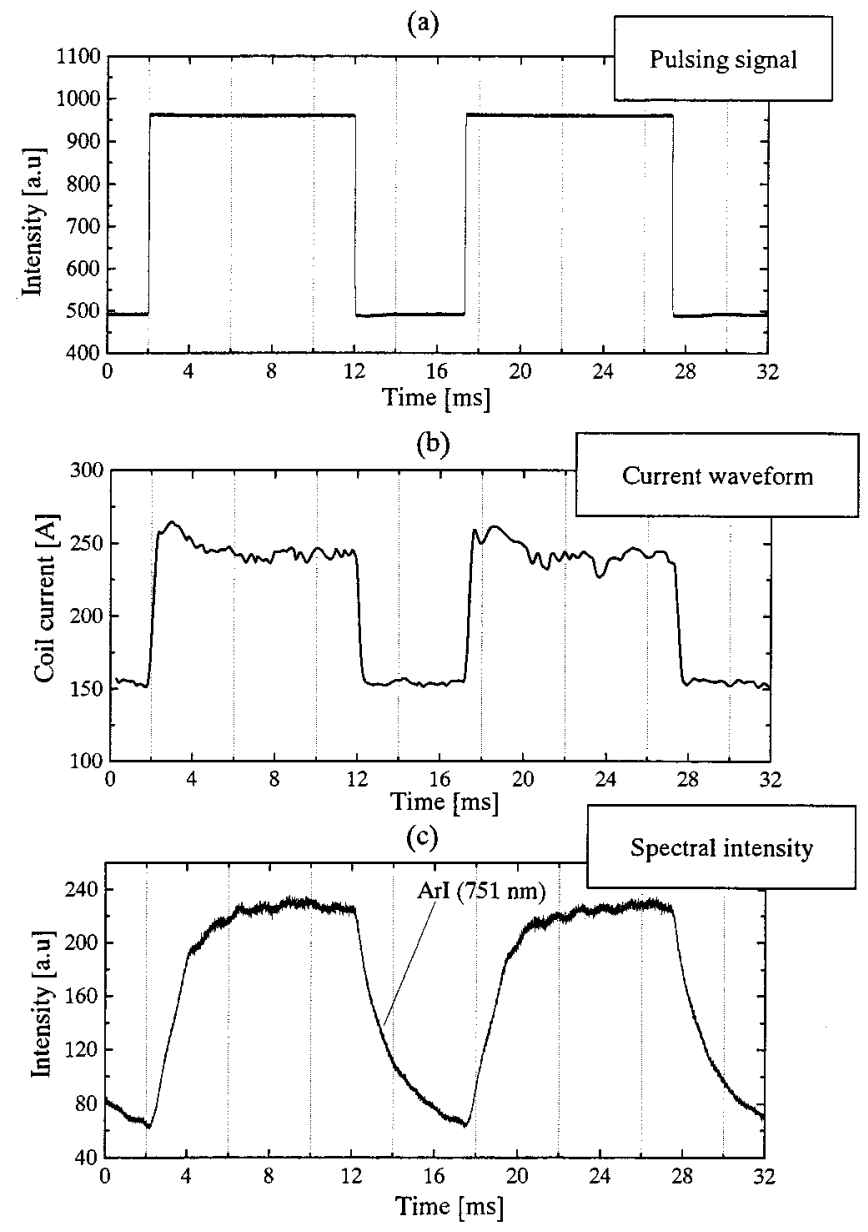

Fig. 3. Spectroscopically measured time-dependent (a) pulsed signal intensity, (b) magnitude of coil current, and (c) radiation intensity of atomic argon line $(751 \mathrm{~nm})$ at $10 \mathrm{~mm}$ below the downstream coil $(z=223 \mathrm{~mm})$.

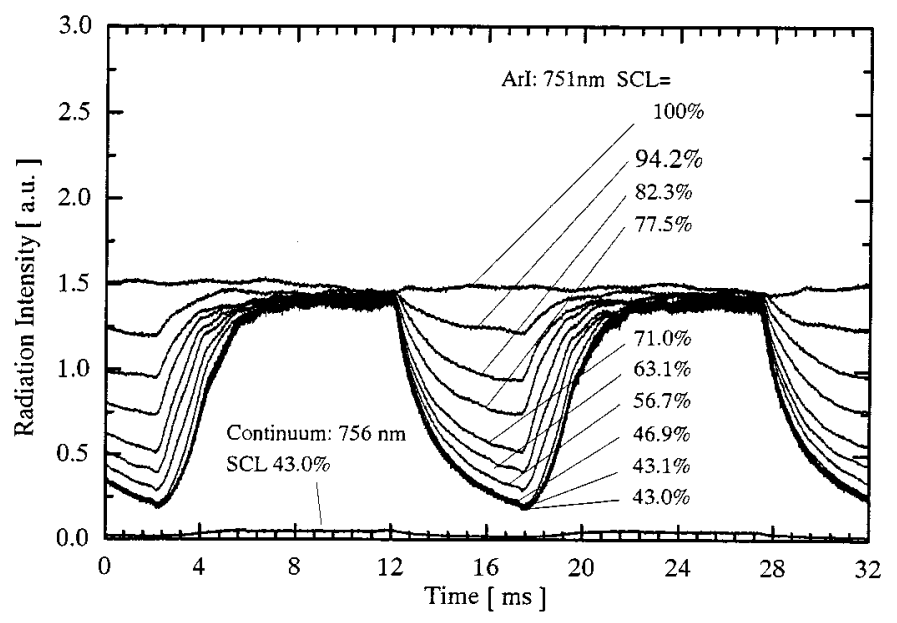

Fig. 4. SCL effect upon the temporal radiation intensity at $10 \mathrm{~mm}$ below the downstream coil $(z=223 \mathrm{~mm})$.

presented in Fig. 5(a) and (b), respectively. The figure shows that the intensities at $r=17.5 \mathrm{~mm}$ are stronger than the intensity at the other two radial positions. Axisymmetric thermal plasmas are typically strong around the middle from the axis of symmetry $(r=0)$ and wall. This is the obvious reason for the stronger spectral intensity at $r=17.5 \mathrm{~mm}$. However, the
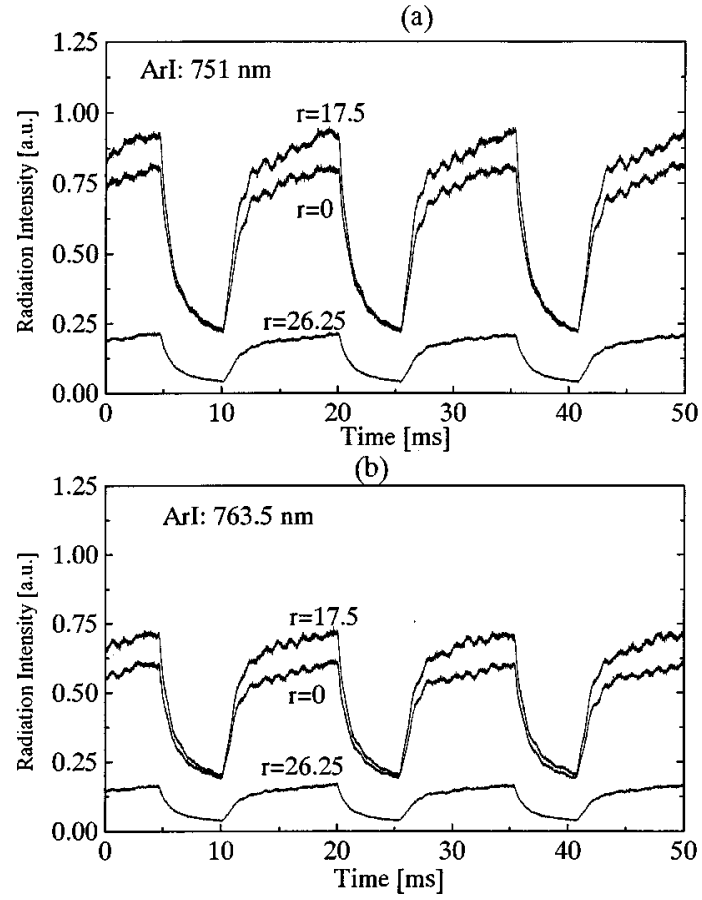

Fig. 5. Time-dependent change of spectral intensity at three radial positions $(r=0,17.5$ and $26.25 \mathrm{~mm})$ at mid-coil $(z=136.5 \mathrm{~mm})$ for (a) $751-\mathrm{nm}$ line and (b) for 763.5-nm line.

intensity profiles are of similar pattern for the both lines. The response of spectral intensity is found faster at $r=17.5 \mathrm{~mm}$ than those of the other two positions, and it is significantly slow at $r=26.25 \mathrm{~mm}$. For instance, the response time of intensity at $r=17.5 \mathrm{~mm}$ is found as $3.17 \mathrm{~ms}$, whereas, it is 4.5 and $5.89 \mathrm{~ms}$ at $r=0$, and $26.25 \mathrm{~mm}$, respectively. Thus, it can be inferred that pulsed plasma does not respond uniformly over the spatial positions, and the response is slower in the cooler region of plasma. It has been realized by researchers [24] that cooler region of plasma usually stays at the non-LTE condition, indicating a possible interlink between the slower response and the non-LTE situation of cooler plasma.

Fig. 6 depicts the comparison between the time-dependent spectral intensities at 751 and $763.5 \mathrm{~nm}$ for three radial positions: Fig.6(a) $r=0$ (on the axis of symmetry), Fig.6(b) $r=17.5 \mathrm{~mm}$, and Fig.6(c) $r=26.25 \mathrm{~mm}$. The 751-nm line is stronger for any radial position than the other, but both of them respond almost at the same phase with respect to the change in operating conditions. Thus, selection of the spectral line does not effect the determination of plasma properties. Therefore, our numerical part is arranged for the 751-nm line only, and it eventually represents cumulative plasma properties.

\section{Time-Dependent Mathematical Model}

The numerical model is based on [18]. The time-dependent conservation equations of mass, momentum, and energy along with the vector potential form of Maxwell's equations are solved simultaneously.

\section{A. Assumptions}

It was assumed that the plasma flow is 2-D and axi-symmetric, laminar and incompressible, the ICP is in the state of 
(a)

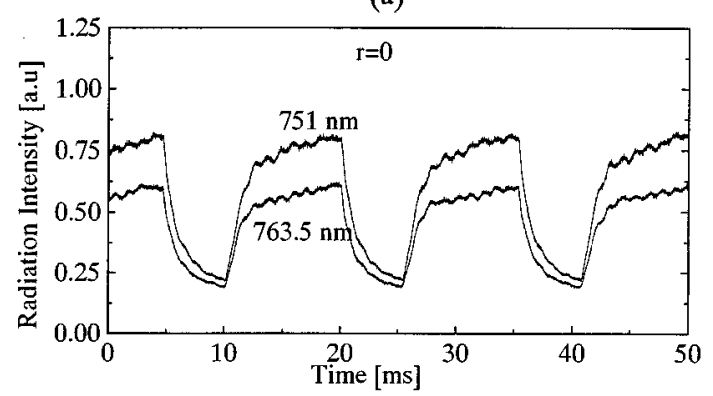

(b)

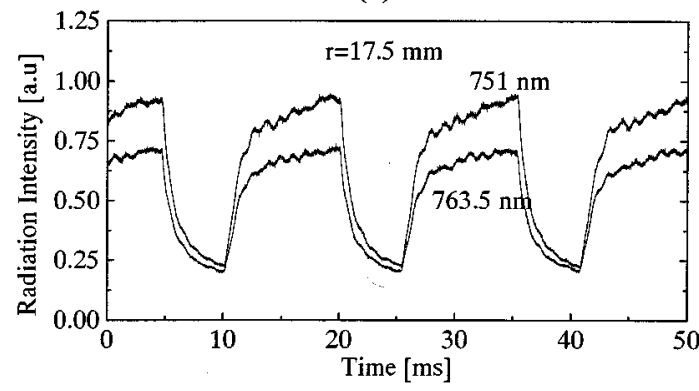

(c)

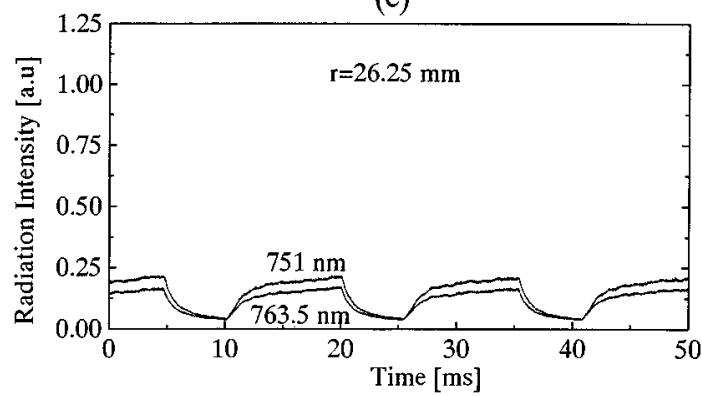

Fig. 6. Comparison between the spectral intensities of 751-nm and 763.5-nm lines at mid-coil $(z=136.5 \mathrm{~mm})$ for three radial positions: (a) $r=0$, (b) $r=17.5 \mathrm{~mm}$, and (c) $r=26.25 \mathrm{~mm}$.

local thermodynamic equilibrium (LTE), and optically thin. Furthermore, viscous dissipation and displacement currents were assumed to be negligible, and each coil turn was considered to be planar.

\section{B. Computational Procedure}

The governing equations, which are listed in Appendix A, were solved numerically using the SIMPLER algorithm of Patankar [21]. The algorithm is based on a control-volume finite-difference scheme for solving the transport equations of incompressible fluids. Calculations were performed for a 35 (in $r$ direction) by 93 (in $z$ direction) nonuniform grid system.

\section{Thermophysical Properties}

Thermodynamic and transport properties of argon at atmospheric pressure were obtained from [22], and the radiation loss data from [23]. It was assumed that the effect of pressure on thermal and electrical conductivities, specific heat at constant pressure, and viscosity is negligible. Density and volumetric radiation losses were assumed to vary linearly with pressure.

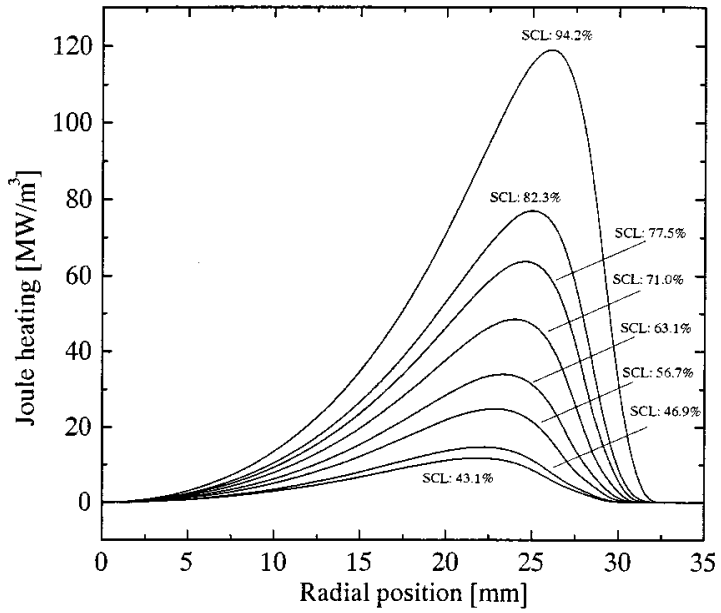

Fig. 7. SCL effect upon the radial distribution of Joule heating density at mid-coil $(z=136.5 \mathrm{~mm})$ and at the end of off-time.

\section{Results and Discussions}

The numerical calculations are done for a 10-turns coil and $80 \mathrm{~L} / \mathrm{m}$ gas flow rate, keeping all other operating conditions the same to Table I. Here, the spectral intensity of a 751-nm argon line is calculated along-with other plasma properties, and the predicted intensity thus obtained is compared with the corresponding experimental intensity. Using the power profile program of the ICP code, the coil current was first determined corresponding to the active plasma power, and this was found to be $145 \mathrm{~A}$. For the $30-\mathrm{kW}$ forward power, we assumed that the active plasma power would be $25.5 \mathrm{~kW}$, which is $85 \%$ of the supply power. Note that the inverter power supply has a good matching efficiency of more than $85 \%$. The coil current for $25.5 \mathrm{~kW}$ plasma power is corresponding to the upper level current of the pulsed signal and it is dropped appropriately to make the desirable SCL.

Induced effect of externally applied magnetic field on plasma fields is naturally strong around the mid-coil region. So, the SCL effect upon the radial distribution of the Joule heating density at mid-coil is determined and presented in Fig. 7. Plasma dissipation is the maximum around $r=22 \mathrm{~mm}$, which is within the skin-depth region of plasma [1]. The figure also infers that lowering of SCL directly decreases the amount of forwarded, as well as active, plasma power. For 94.2\% SCL, the maximum value of the Joule heating density is predicted as $118.74 \mathrm{MW} / \mathrm{m}^{3}$, and it is $11.81 \mathrm{MW} / \mathrm{m}^{3}$ for $43.1 \% \mathrm{SCL}$.

The effect of SCL upon the temperature at mid-coil $(z=$ $136.5 \mathrm{~mm}$ ) is shown in Fig. 8 for two radial positions: (a) on the axis of symmetry ( $r=0)$ and (b) at $r=17.5 \mathrm{~mm}$. The temperature change became stronger with the decrease of SCL at both radial positions because of stronger change of operating condition via lowered SCL. However, the on-time responses were different in nature at these two radial positions: the temperature on the axis of symmetry is found to be larger around the last $4 \mathrm{~ms}$ of the on-time for lower SCL but it attains more or less similar magnitude around the ending-duration of on-time at $r=17.5 \mathrm{~mm}$. The temperature decreased gradually with the lowering of SCL during off-time for both radial positions. 
(a)

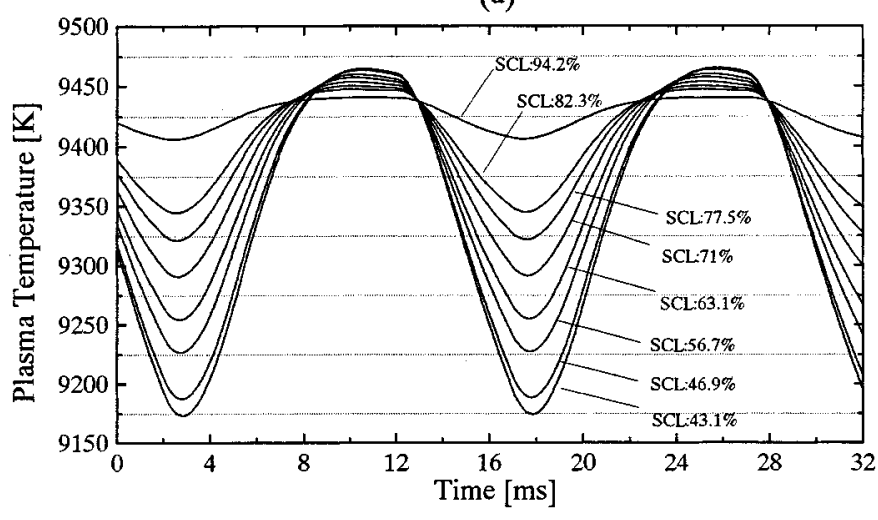

(b)

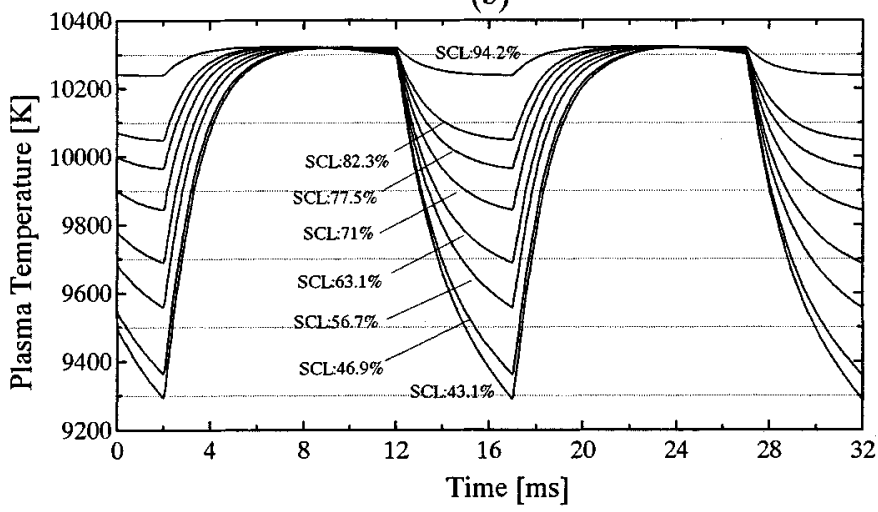

Fig. 8. SCL effect upon the time-dependent temperature at mid-coil $(z=$ $136.5 \mathrm{~mm}$ ) and (a) $r=0$ and (b) $r=17.5 \mathrm{~mm}$.

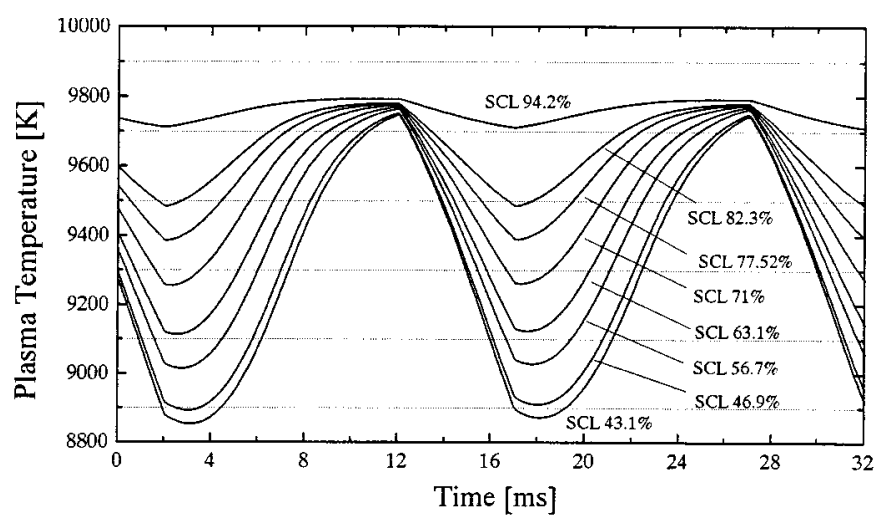

Fig. 9. SCL effect upon the time-dependent temperature at $10 \mathrm{~mm}$ below the downstream coil $(z=223 \mathrm{~mm})$ and $r=17.5 \mathrm{~mm}$.

Since plasma response is profound within the skin-depth region of plasma, temperature fields are found to respond rapidly and strongly at $r=17.5 \mathrm{~mm}$ (which is within the skin-depth region). A similar set of temporal temperature distribution is shown in Fig. 9 for $z=223 \mathrm{~mm}(10 \mathrm{~mm}$ below the downstream coil) and $r=17.5 \mathrm{~mm}$. In nature, the effect of SCL was the same as the one discussed following Fig. 8(b). However, the temperature did not attain a similar value during the on-time, rather decreased gradually with the decrease of SCL. And the response of temperature was slower compared to the case noticed at mid-coil $(z=136.5 \mathrm{~mm})$. The externally applied magnetic field through the load coil is the input to the plasma and any change of this field will transfer rapidly to plasma-region

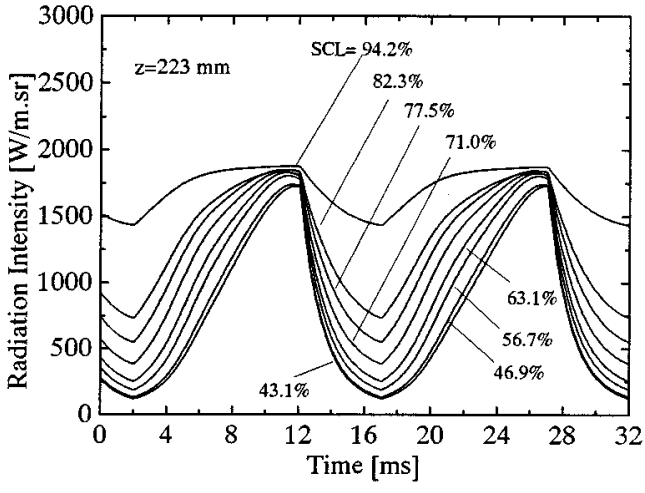

Fig. 10. SCL effect upon the temporal radiation intensity of atomic argon line $(751 \mathrm{~nm})$ at $10 \mathrm{~mm}$ below the downstream coil $(z=223 \mathrm{~mm})$.

surrounded by the coil compared to the other parts of plasma. That's why the temporal temperature at mid-coil, around where the externally applied fields are stronger, is found to respond rapidly than at $10 \mathrm{~mm}$ below the downstream coil. The weak plasma at $z=223 \mathrm{~mm}$ can not reach a constant temperature during on-time due to characteristic inertia prevails in the weak plasma.

\section{COMPARISON BETWEEN EXPERIMENTAL AND THEORETICAL RESULTS}

The integrated argon line intensity at a particular axial position can be calculated numerically by the following relation:

$$
I_{z}=2 \pi \int_{0}^{R_{0}} I(T, r) d r
$$

where

$$
I(T, r)=C \frac{N(T)}{Z(T)} e^{-E_{n} / k T} .
$$

Here, $I(T, r)$ is the temperature-dependent spectral intensity for a particular radial position; $C=h c v g_{n} A_{n} / 4 \pi$, is to account all constants; $N(T)$ and $Z(T)$ are the temperature-dependent particle density and internal partition function, respectively; $E_{n}$ is the energy of the level; and $k$ is the Boltzmann's constant.

The numerically obtained intensity of the Ar line at $751 \mathrm{~nm}$ is illustrated in Fig. 10 for the axial position of $10 \mathrm{~mm}$ below the downstream coil $(z=223 \mathrm{~mm})$. This figure illustrates the SCL effects upon the calculated intensities and is comparable with the experimental results of Fig. 4. As found in experiment, on-time response becomes a little slower with the decrease of SCL. The intensities measured experimentally and calculated theoretically for $63.1 \%$ SCL are compared in Fig. 11. Both the experimental and theoretical results change magnitudes at almost the same order $(1.41 / 0.39=3.6$ in experiment; $5.58 / 1.7=3.3$ in theory) with pulsation. One major discrepancy found between these two results is that the rising time of the numerically predicted intensity is longer than that of the experimentally measured intensity. This discrepancy would be due to the LTE assumption in the model. It is an understanding that plasma deviates from the LTE condition even at a high (atmospheric) pressure [24], significantly around the cooler region of plasma. During the end of off-time, plasma temperature drops 


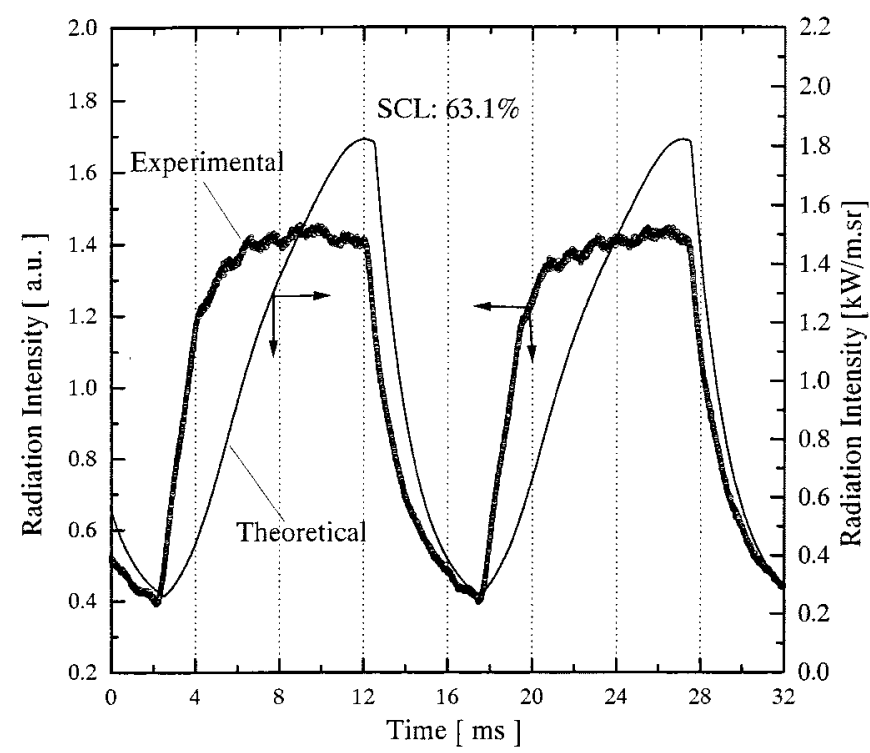

Fig. 11. Comparison between the measured and calculated radiation intensities for $63.1 \% \mathrm{SCL}$.

to a lower value due to insignificant power input, as well as due to the cooling effect by the cold gas-flow and, thus, the plasma would be in non-LTE condition. Nevertheless, the introduction of power by the on-pulsation inadvertently deviates plasma from LTE condition. There is a physical understanding that the supplied energy to plasma is absorbed by the electrons first, and this energy is then transmitted to all other species through collisions between electrons and other species until energy balance is reached. For this reason, it is assumed that electron temperature is, at least, initially higher than the heavy particle temperature. Under these circumstances, the radiation intensity (which is basically governed by the electron temperature of plasma) of non-LTE plasma responds faster practically than the theoretical one of LTE plasma. It can also be attributed that both the experimental and theoretical responses of plasma during off-time are almost same. Around the end of on-period, plasma at high temperature stays almost into LTE condition, and hence, the plasma responses for both experimental and theoretical cases are alike.

Response times are determined, both experimentally and theoretically, from the spectral intensities for both on- and offtimes. The definitions of these response times are illustrated clearly in Fig. 12. The response time for on-time is the time required by the intensity to reach from 10\%-90\% level of the maximum value, and for off-time, it is the time required by the intensity to reach $90 \%$ to $10 \%$ level of the minimum value. Fig. 13 shows the SCL effect upon the response times: Fig.13(a) for on-time and Fig.13(b) for off-time. Response times for on-time pulsation are, in general, higher (about double) in theoretical predictions than those found in experimental determinations. But for off-time pulsation, the theoretically predicted response times are almost equal to the values found from experimental determinations. However, the trends in change of response times with the SCL are found identical in both experiment and theory for both pulsations. The stronger nonequilibrium effects of practical plasma around on-pulsation, and near-about equilibrium effect around off-pulsation, are the reasonable interpretations for the predicted nature of response times.

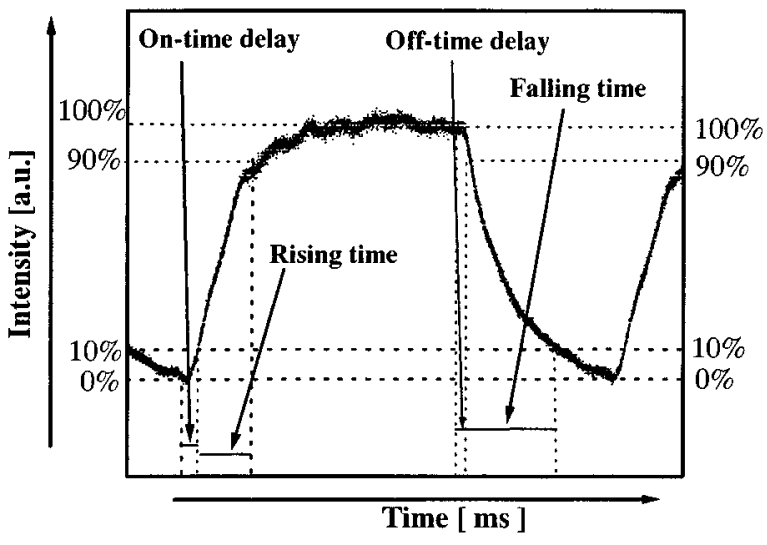

Fig. 12. Definitions of response times for both on- and off-pulsations.
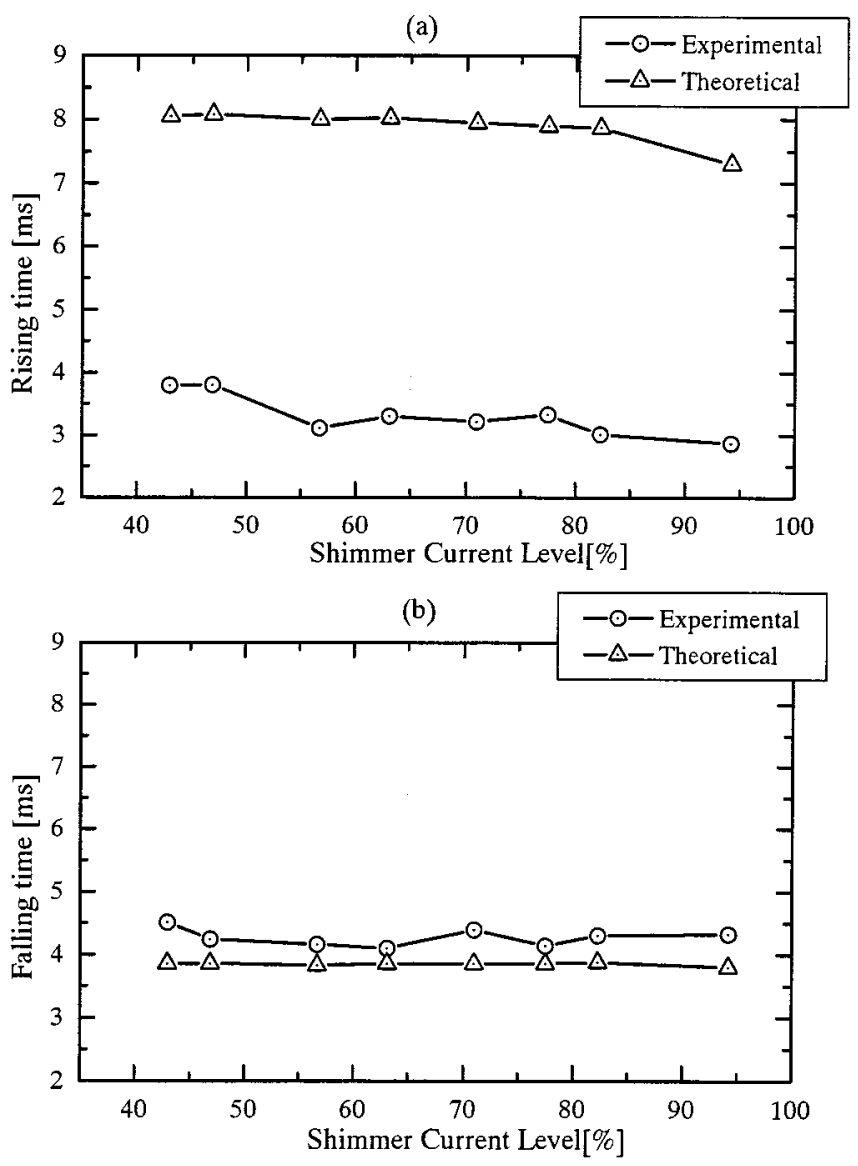

Fig. 13. SCL effect upon experimental and theoretical response times and comparison between them: (a) for on-time and (b) for off-time.

\section{CONCLUSION}

Experimental diagnosis was carried out for an ICP system working with a MOSFET-employed inverter power supply and a torch of very long coil length $(153 \mathrm{~mm})$. The plasma under pulsing mode was generated with an 80 or $90 \mathrm{~L} / \mathrm{m}$ of argon flow-rate through the sheath channel. For the same operating conditions, a two-dimensional time-dependent LTE model of ICP code was solved to predict temporal behavior of plasma fields and to compare with the experimental measurements. The findings can be summarized as follows. 
1) The plasma power, as well as the plasma temperature, can be controlled according to necessity in time domain by changing the SCL or the lower value of coil current.

2) There is a limit of SCL below of which plasma disappears for other particular operating conditions; it is $43.1 \%$ at 80 $\mathrm{L} / \mathrm{m}$ argon flow rate for this specific ICP torch with the operating conditions listed in Table I.

3) Plasma properties by 751- and 763.5-nm argon lines are seen similar so that line selection would not effect the plasma diagnosis.

4) Plasma is found to respond a bit slowly after on-time at lower SCL, and it is the additional time needed by the decreased temperature (of off-time) to attain the temperature level corresponding to the higher SCL temperature at the end of off-time. And the SCL effect upon plasma response after off-pulsation is negligible because the plasma stays about similar condition by the similar coil current level just before off-pulsation.

5) Plasma usually deviates from LTE, especially around the on-time, and the degree of deviation depends on SCL. Therefore, the response times for on-pulsation, predicted by the LTE model, are found to be longer than those of practical plasma due to non-LTE situation of plasma, as well as SCL effect mentioned in the last item.

\section{APPENDIX A \\ BASIC TIME-DEPENDENT EQUATIONS}

The time-dependent conservation equations for the transport of mass, momentum and energy are as follows:

- conservation of mass

$$
\frac{1}{\rho} \frac{D \rho}{D t}+\nabla \cdot \mathbf{u}=0
$$

- conservation of momentum

1) $r$-momentum

$$
\begin{aligned}
\rho \frac{D v}{D t}=-\frac{\partial p}{\partial r}+\nabla \cdot \mu \nabla v & +\frac{1}{r} \frac{\partial}{\partial r}\left(\mu r \frac{\partial v}{\partial r}\right)+\frac{\partial}{\partial z}\left(\mu \frac{\partial u}{\partial r}\right) \\
& -\frac{2 \mu v}{r^{2}}+\frac{\rho w^{2}}{r}+(\mathbf{j} \times \mathbf{B})_{r}
\end{aligned}
$$

2) $z$-momentum

$$
\begin{aligned}
\rho \frac{D u}{D t}=-\frac{\partial p}{\partial z}+\nabla \cdot \mu \nabla u & +\frac{1}{r} \frac{\partial}{\partial r}\left(\mu r \frac{\partial v}{\partial z}\right) \\
& +\frac{\partial}{\partial z}\left(\mu \frac{\partial u}{\partial z}\right)+(\mathbf{j} \times \mathbf{B})_{z}
\end{aligned}
$$

3) $\theta$-momentum

$\rho \frac{D w}{D t}=\nabla \cdot \mu \nabla w-\frac{\rho v w}{r}-\frac{1}{r} \frac{\partial(\mu w)}{\partial r}+\mu \frac{\partial}{\partial r}\left(\frac{w}{r}\right)$

- conservation of energy

$$
\rho \frac{D h}{D t}=\nabla \cdot \kappa \nabla T+\mathbf{j} \cdot \mathbf{E}-R^{\circ}
$$

where

$D / D t \equiv \partial / \partial t+\mathbf{u} . \nabla$ substantial derivative;

$\nabla \quad$ gradient operator in cylindrical coordinates;

$\rho \quad$ plasma density;

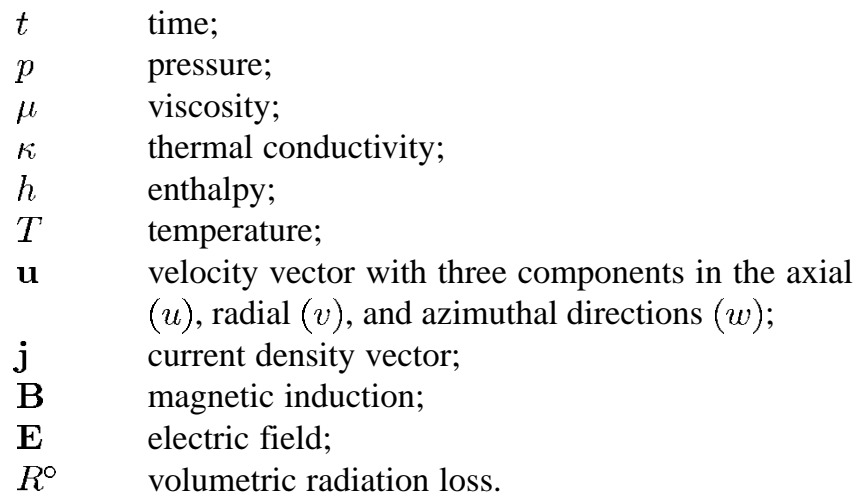

The electromagnetic fields and current density were calculated from the vector potential form of the Maxwell's equations. The vector potential, $\mathbf{A}(\mathbf{r}, t)$, is defined as

$$
\mathbf{B}=\mu_{0} \mathbf{H} \equiv \nabla \times \mathbf{A}
$$

where

H magnetic field intensity;

$\mathbf{r}$ position vector;

$\mu_{0}=4 \pi \times 10^{-7}(\mathrm{H} / \mathrm{m})$ permeability of free space.

For a standard induction plasma torch with conventional coil geometry (as seen in Fig. 1), it is reasonable to assume that the electric field and, thus, the vector potential has only tangential component [9]. The electric field $\left(E_{\theta}\right)$, axial and radial components of magnetic field $\left(H_{z}\right.$ and $\left.H_{r}\right)$, and vector potential $\left(A_{\theta}\right)$ are co-related as follows:

$$
E_{\theta}=-i \varpi A_{\theta} \quad \mu_{0} H_{z}=\frac{1 \partial}{r \partial r}\left(r A_{\theta}\right) ; \quad \mu_{0} H_{r}=-\frac{\partial A_{\theta}}{\partial z} .
$$

The Joule heating and axial and radial Lorentzian forces are written as

$$
\begin{aligned}
\dot{P} & =\frac{1}{2} \sigma\left[E_{\theta} E_{\theta}^{*}\right] \\
F_{z} & =\frac{1}{2} \mu_{0} \sigma \Re\left[E_{\theta} H_{r}^{*}\right] \\
F_{r} & =\frac{1}{2} \mu_{0} \sigma \Re\left[E_{\theta} H_{z}^{*}\right]
\end{aligned}
$$

where $r, \theta$, and $z$ are the radial, tangential, and axial coordinates, respectively, and $\Re$ is the real component.

\section{APPENDIX B BOUNDARY CONDITIONS}

The boundary conditions for conservation equations of mass, momentum, and energy [(A.1)-(A.3)] were: at the inlet, gas temperature was given as $300 \mathrm{~K}$ and uniform velocity profiles were assumed based on the given flow rates; on the axis of symmetry, the symmetry conditions were imposed; on the walls, the no-slip condition was assumed, and the outer wall temperature was given as $350 \mathrm{~K}$; and, at the exit, axial gradients were set equal to zero.

The vector potential is zero on the axis of symmetry since the electric field is zero. At the inlet, exit, and the wall of the torch, the following boundary condition was assumed:

$$
A_{\theta}(r, t)=\frac{\mu_{0}}{4 \pi} \int_{\text {Vol. }} \frac{j\left(r^{\prime}, t\right)}{\left|r-r^{\prime}\right|} d^{3} r^{\prime}
$$


where the integral extends over all the current carrying regions in space, including the coil. Equation (B.1) was further simplified and was written explicitly in terms of the complete elliptic integrals [20].

\section{REFERENCES}

[1] K. C. Paul, T. Takashima, and T. Sakuta, "Copper vapor effect on $\mathrm{RF}$ inductively coupled $\mathrm{SF}_{6}$ plasmas," IEEE Trans. Plasma Sci., pp. 1000-1009, June 1998.

[2] K. C. Paul et al., "Diagnosis of inductively coupled $\mathrm{Sf}_{6}$ and $\mathrm{N}_{2}$ plasmas at atmospheric pressure," Thin Solid Films, vol. 345, pp. 134-139, 1999.

[3] K. C. Paul, "Fundamental plasma studies for different gases in the phase of circuit breaker applications," Ph.D. dissertation, Dept. Elect. Comput. Eng., Kanazawa Univ., Kanazawa, Japan, 1998.

[4] P. Meubus, "Transient conditions in inductively heated plasmas: Thermodynamic equilibrium and temperature measurements," Can. J. Phys., vol. 60, pp. 886-892, 1982.

[5] J. M. de Regt, J. A. M. van der Mullen, and D. C. Schram, "Responses of the electron density and temperature to the power interruption measured by Thompson scattering in an inductively coupled plasma," Phys. Rev, vol. 52, pp. 2982-2987, 1995.

[6] F. H. A. G. Fey et al., "Instantaneous and delayed responses of line intensities to interruption of the RF power in an argon inductively coupled plasma," Spectrochim. Acta, vol. 46B, pp. 885-900, 1991.

[7] T. Sakuta et al., "Experimentally diagnosed transient behavior of pulse modulated inductively coupled thermal plasma," J. Appl. Phys., vol. 85, pp. 1372-1377, 1999.

[8] T. Ishigaki et al., "Generation of pulse-modulated induction thermal plasma at atmospheric pressure," Appl. Phys. Lett., vol. 71, pp. 3787-3789, 1997.

[9] J. Mostaghimi and M. I. Boulos, "Two-dimensional electromagnetic field effects in induction plasma modeling," Plasma Chem. Plasma Process., vol. 9, pp. 25-44, 1989.

[10] M. Cai et al., "Computer simulation of argon-nitrogen and argon-oxygen inductively coupled plasmas," Spectrochim. Acta, vol. 52B, pp. 369-386, 1997.

[11] J. W. McKelliget and N. El-Kaddah, "The effect of coil design on materials synthesis in an inductively coupled plasma torch," J. Appl. Phys., vol. 64, pp. 2948-2954, 1988.

[12] J. Mostaghimi and M. I. Boulos, "Effect of frequency on local thermodynamic equilibrium conditions in an inductively coupled argon plasma at atmospheric pressure," J. Appl. Phys., vol. 68, pp. 2643-2648, 1990.

[13] M. Cai, A. Montaser, and J. Mostaghimi, "A two-temperature model for the simulation of atmospheric pressure helium ICP's," Appl. Spectrosc., vol. 49, pp. 1390-1402, 1995.

[14] J. Kim, J. Mostaghimi, and R. Iravani, "Performance analysis of a radio frequency induction plasma generator using nonlinear state-space approach," IEEE Trans. Plasma Sci., vol. 25, pp. 1023-1028, Oct. 1997.

[15] T. Sakuta et al., "Effects of plasma diameter and operating frequency on dynamic behavior of induction thermal plasma," Plasma Sources Sci. Technol., vol. 2, pp. 67-71, 1993.

[16] T. Sakuta, "Dynamic behaviors and stability of induction thermal plasma," in Proc. 3rd Asia-Pacific Conf. Plasma Science. and Technology., vol. 2, Tokyo, Japan, 1996, pp. 385-390.

[17] T. Suekane et al., "Numerical studies on the nonequilibrium inductively coupled plasma with metal vapor ionization," IEEE Trans. Plasma Sci., vol. 24, pp. 1147-1154, June 1996.

[18] J. Mostaghimi, K. C. Paul, and T. Sakuta, "Transient responses of the radio frequency inductively coupled plasma to a sudden change in power," J. Appl. Phys., vol. 83, pp. 1898-1908, 1998.

[19] K. C. Paul et al., "Diagnosis of pulse modulated $\mathrm{Ar}-\mathrm{H}_{2}$ and $\mathrm{Ar}-\mathrm{N}_{2}$ plasmas at atmospheric pressure," in Proc. 14th Int. Symp. Plasma Chemistry, vol. 1, Prague, Czech Republic, Aug. 1999, pp. 179-184.

[20] J. D. Jackson, Classical Electrodynamics. New York: Wiley, 1962.

[21] S. V. Patankar, Numerical Fluid Flow and Heat Transfer. New York: Hemisphere, 1980.

[22] M. I. Boulos, P. Fauchais, and E. Pfender, Thermal Plasmas-Fundamentals and Applications. New York: Plenum, 1994, vol. 1.

[23] D. L. Evans and R. S. Tankin, "Measurement of emission and absorption of radiation by an argon plasma," Phys. Fluids, vol. 10, pp. 1137-1144, 1967.

[24] J. Mostaghimi, P. Proulx, and M. I. Boulos, "A two-temperature model of the inductively coupled R.F. plasma," J. Appl. Phys., vol. 61, pp. 1753-1760, 1987.

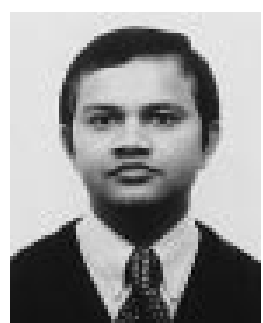

K. C Paul (M'99) was born in 1965. He received the B.S. degree from the Department of Electrical and Electronic Engineering, Bangladesh Institute of Technology, Chittagong, Bangladesh, and the M.Eng. and D.Eng. degrees from Kanazawa University, Kanazawa, Japan, in 1990, 1995, and 1998, respectively.

He was a Japan Society for the Promotion of Science Post-Doctoral Research Fellow with Kanazawa University from April 1998 to March 2000. Presently, he is a Research Fellow in the Electrical and Computer Engineering Department, Toronto University, Toronto, ON, Canada. His fields of interest include RF-ICP's, circuit breakers, and dc lamp arcs.

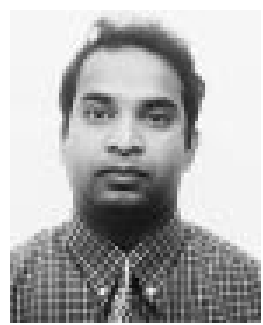

M. M. Hossain (M'01) was born in December 1, 1969. He received the B.S. degree in 1994 from Bangladesh University of Engineering and Technology, Dhaka, Bangladesh, and the M.S. degree in electrical and electronic engineering in 2000 from Kanazawa University, Kanazawa, Japan, where he is currently working toward the Ph.D degree in deputation.

Since April 1994, he has been a Lecturer in the Department of Electrical and Electronic Engineering, Bangladesh Institute of Technology, Chittagong, Bangladesh, where he recently became an Assistant Professor. His field of research is radio frequency induction thermal plasma for plasma processing.

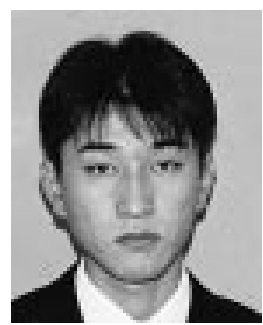

Y. Hashimoto was born in 1975 . He received the B.S. and M.S. degrees in electrical and electronic engineering from Kanazawa University, Kanazawa, Japan, in 1998 and 2000, respectively.

Currently, he is with Nippon Telegraph and Telephone Corporation, Tokyo, Japan. His research interests include radio-frequency inductively coupled plasma.

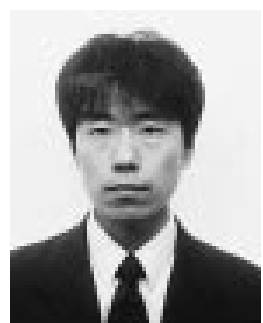

Y. Tanaka was born in Japan in 1970. He received the B.S., M.S. and Ph.D. degrees in electrical engineering from Nagoya University, Nagoya, Japan, in 1993, 1995 and 1998, respectively.

Since 1998, he has been a Research Associate in the Department of Electrical and Electronic Engineering, Kanazawa University, Kanazawa, Japan. His research interests include the arc interruption phenomena, and thermal plasma applications.

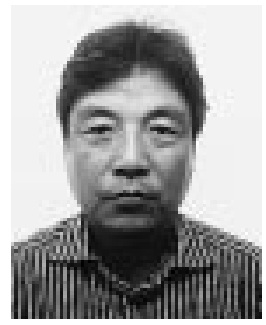

Tadahiro Sakuta (M'91) was born in $1950 . \mathrm{He}$ received the $\mathrm{Ph} . \mathrm{D}$ degree in electrical engineering from the Department of Electrical Engineering, Nagoya University, Nagoya, Japan, in 1980.

In April 1981, he was appointed as a Research Associate in the Department of Electrical Engineering, Nagoya University. Since April 1988, he has been with the Department of Electrical and Electronic Engineering, Kanazawa University, Kanazawa, Japan, first as an Associate Professor and currently as a Professor. His research interests include the diagnosis and application of high-pressure thermal plasma, including inductively coupled plasmas and circuit breaker arcs.

Dr. Sakuta is a member of the Society of Applied Physics and Institute of Electrical Engineers of Japan. 